\title{
Today's psychiatric neuroscience advances were science fiction during my residency
}

\section{During my residency training years, I had many rosy and bold dreams about the future of psychiatry, hoping for many breakthroughs.}

Early on, I decided to pursue an academic career, and specifically to focus on the neurobiology of schizophrenia, bipolar disorder, and other psychoses. I secured a neuroscience mentor, conducted a research project, and presented my findings at the American Psychiatric Association Annual Meeting. Although at the time everyone used the term "functional" to describe mental illnesses, I was convinced that they were all neurologic conditions, with prominent psychiatric manifestations. And I have been proven right.

After my residency, I eagerly pursued a neuroscience fellowship at the National Institutes of Health. My fantasy was that during my career as a psychiatric neuroscientist, brain exploration would uncover the many mysteries of psychiatric disorders. I was insightful enough to recognize that what I envisioned for the future of psychiatry qualified as science fiction, but I never stopped dreaming.

Today, the advances in psychiatric neuroscience that were unimaginable during my residency have become dazzling discoveries. My journey as a psychiatric neuroscientist has been more thrilling than I ever imagined. I recall doing postmortem research on the brains of hundreds of deceased psychiatric patients, noticing sulci widening and ventricular dilatation, and wondering whether one day we would be able to detect those atrophic changes while the patients were alive. Although I measured those changes in postmortem brains, I was cognizant that due to preservation artifacts, such measurements were less reliable than measurements of living brains.

And then the advent of neuroimaging fulfilled my fantasies. This began towards the end of my fellowship, and has exploded with neurobiologic findings throughout my academic career. Then came dramatic methodologies to probe brain molecular and cellular pathologies, followed by breakthrough clinical advances. Entirely new vistas of research into psychiatric brain disorders are opening every day. The exhilaration will never end!

\section{From science fiction to clinical reality}

Here is a quick outline of some of the "science fiction" of psychiatry that has come true since my training days. Back then, these discoveries were completely absent from the radar screen of psychiatry, when it was still a fledgling medical

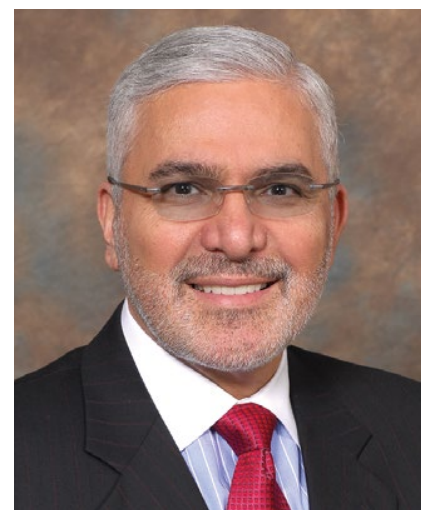

Henry A. Nasrallah, MD Editor-in-Chief

doi: 10.12788/cp.0116

\section{Advances in psychiatric neuroscience that were unimaginable during my residency have become dazzling discoveries}




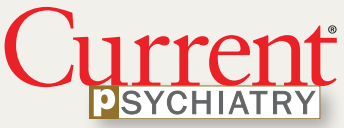

Editorial Staff

EDITOR Jeff Bauer

SENIOR EDITOR Sathya Achia Abraham

ASSISTANT EDITOR Jason Orszt

WEB EDITOR Kathryn Wighton

Art \& Production Staff

CREATIVE DIRECTOR Louise Koenig

ART DIRECTOR Pat Fopma

DIRECTOR, JOURNAL MANUFACTURING

Michael Wendt

PRODUCTION MANAGER Donna Pituras

Publishing Staff

PUBLISHER Sharon Finch

DIRECTOR EBUSINESS DEVELOPMENT

Alison Paton

SENIOR DIRECTOR OF SALES

Tim LaPella

Editor-in-Chief Emeritus

James Randolph Hillard, MD

Frontline Medical Communications

VP, SALES Mike Guire

VP, DIGITAL CONTENT \& STRATEGY

Amy Pfeiffer

PRESIDENT, CUSTOM SOLUTIONS

JoAnn Wahl

CIRCULATION DIRECTOR Jared Sonners

DIRECTOR, CUSTOM PROGRAMS

Patrick Finnegan

In affiliation with Global Academy for

Medical Education, LLC

PRESIDENT David J. Small, MBA

\section{FRONTLINE MLedge}

7 Century Drive, Suite 302

Parsippany, NJ 07054

Tel: (973) 206-3434

Fax: (973) 206-9378

www.frontlinemedcom.com

Subscription Inquiries:

subscriptions@mdedge.com

Published through an

educational partnership with

6 Current Psychiatry

April 2021 specialty struggling to emerge from the dominant yet nonempirical era of psychoanalysis.

Brain exploration methods. Unprecedented breakthroughs in computer technology have allowed psychiatric neuroscientists to create a new field of neuroimaging research that includes:

- cerebral blood flow (CBF)

- position emission tomography (PET)

- single photon emission computed tomography (SPECT).

These functional neuroimaging methods (using ionizing radiation) have enabled clinicians to see abnormal blood flow patterns in the brains of living patients. One of the earliest findings was hypofrontality in patients with schizophrenia, implicating frontal pathology in this severe brain disorder. PET was also used for dopamine and serotonin receptor imaging.

Computerized axia tomography. Compared with skull X-rays, CT ("CAT") scans provided a more detailed view of brain tissue, and began a structural neuroimaging revolution that enriched psychiatric research, but also was applied to organs other than the brain.

Magnetic resonance imaging (MRI) became the "big kahuna" of neuroimaging when arrived in the early 1980s and quickly supplanted CT research because it is safer (no ionizing radiation, and it can be repeated multiple times with or without tasks). It also provided exquisite neuroanatomical details of brain tissue with stunning fidelity. Subsequently, several MRI techniques/software programs were developed that advanced research in psychiatry to multiple new frontiers, including: MRI

- Morphological neuroimaging with

- Magnetic resonance spectroscopy (MRS), which acts like a living, noninvasive biopsy of several chemicals (such as choline, lactate, glutamine, adenosine triphosphate, and the neuronal marker $\mathrm{N}$-acetylcysteine) in a small volume $(\leq 1 \mathrm{cc})$ of neural tissue in various regions

- Functional MRI (fMRI), which measures blood flow changes during actual or imagined tasks in the brains of patients vs healthy controls

- Diffusion tensor imaging (DTI), which evaluates the integrity of white matter $(60 \%$ of brain volume, including 137,000 miles of myelinated fibers) by measuring the flow of water inside myelinated fibers (anisotropy and diffusivity). DTI of the corpus callosum, the largest brain commissure that is comprised of 200 million interhemispheric fibers, has revealed many abnormalities. This was one of the structures I investigated during my fellowship, including a histopathological study. ${ }^{1}$

All 4 of these neuroimaging techniques continue to generate a wealth of data about brain structure and function in psychosis, mood disorders, anxiety disorders, borderline personality disorder, obsessive-compulsive disorder, eating disorders, and substance use disorders. All these discoveries were utterly impossible to predict during my residency. I am proud to have published the first reports in the literature of ventricular enlargement in patients with bipolar disorder, ${ }^{2}$ cortical atrophy in schizophrenia and mania, reductions of hippocampal volume in patients with schizophrenia using $\mathrm{MRS}^{4}{ }^{4}$ and progressive brain atrophy in patients with schizophrenia. ${ }^{5}$ It is especially gratifying that I played a small role in translating my science fiction fantasies into clinical reality!

Other breakthrough methodologies that are advancing psychiatric neuroscience today but were science fiction during my residency days include: 
- Pluripotent stem cells, which enable the de-differentiation of adult skin cells and then re-differentiating them into any type of cell, including neurons. This allows researchers to conduct studies on any patient's brain cells without needing to do an invasive, high-risk brain biopsy. As a young resident, I would never have predicted that this virtual brain biopsy would be possible!

- Optogenetics, which enables controlling cell behavior using light and genetically encoded light-sensitive proteins. This triggered a cornucopia of neuroscience discoveries by using optogenetics to modulate cell-signaling cascades to understand cellular biology. Halorhodopsin and bacteriorhodopsin are used as tools to turn neurons off or on rapidly and safely.

- Genome-wide association studies (GWAS) have revolutionized the field of molecular neurogenetics and are enabling clinicians to detect risk genes by comparing the DNA samples of thousands of psychiatric patients with thousands of healthy controls. This is how several hundred risk genes have been identified for schizophrenia, bipolar disorder, autism spectrum disorder, and more to come.

- Clustered regularly interspaced short palindromic repeats (CRISPR) is a remarkable genetic "scissors" (that earned its inventors the 2020 Nobel Prize) that allows splicing out a disease gene and splicing in a normal gene. This will have an enormous future application in preventing an adulthood illness at its roots during fetal life. The future medical implications for psychiatric disorders are prodigious!

Clinical advances. Many therapies or approaches that did not exist during my residency (and how I dreamed about them back then!) are available to today's clinicians. These include:

- Rapid-acting antidepressants that reverse severe and chronic depression and suicidal urges within a few hours or a couple of days. As a resident, I waited for weeks or months to see patients with depression reach the full remission that is now achieved practically the same day with IV ketamine, intranasal esketamine, IV scopolamine, and inhalable nitrous oxide. During my residency, the closest thing we had to a rapid-acting treatment for depression was electroconvulsive therapy (ECT), but that usually took 2 to 3 weeks. Psychiatric clinicians should never cease to appreciate how an intractable, treatment-refractory depression can rapidly be turned off like a light switch, restoring normal mood to desperately ill persons.

- Neuromodulation techniques are flourishing. Beyond ECT, transcranial magnetic stimulation (TMS), vagus nerve stimulation (VNS), transcranial direct current stimulation (tDCS), deep brain stimulation (DBS), low field magnetic stimulation (LFMS), magnetic seizure therapy (MST), nearinfrared radiation (NIR), and focused ultrasound (FUS) are approved or under development, offering millions of patients with various neuropsychiatric disorders potential recovery not with pharmacotherapy, but via a braintargeted approach.

- Telepsychiatry. Now taken for granted during the COVID-19 pandemic, telepsychiatry was completely unimaginable during my residency. Yes, we had phones, but not smartphones! The only "zoom" we knew was the furious sound of a sports car engine! To be able to see and evaluate a patient literally anywhere in the world was science fiction personified! Increased remote access to psychiatric care by patients everywhere is a truly remarkable advance that helped avoid a disastrous lack of psychiatric treatment during the current pandemic that brought in-person interactions
To be able to see and evaluate a patient literally anywhere in the world was science fiction personified 


\section{Today, I fully expect that scientific dreams eventually come true through research}

continued from page 7

between psychiatric physicians and their patients to a screeching halt.

- Neurobiologic effects of psychotherapy. Viewing psychotherapy as a neurobiologic treatment was totally unknown and unimaginable during my residency. I was heavily trained in various types of psychotherapies, but not once did any of my supervisors mention experiential neuroplasticity as a brain-altering process, or that psychotherapy changes brain structure, induces experimental neuroplasticity, and induces billions of dendritic spines in patients' cortex and limbic structures, helping them connect the dots and develop new insights. No one knew that psychotherapy can mimic the neural effects of pharmacotherapy.

- Immunomodulatory effects of psychotherapy. It was completely unknown that psychotherapies such as cognitive-behavioral therapy can lower levels of inflammatory biomarkers in patients' CSF and serum. Back then, no one imagined that psychotherapy had immunomodulatory effects. These discoveries are revolutionary for us psychiatrists and confirm the neurobiologic mechanisms of psychotherapy for every patient we treat.

- Epigenetics. This was rarely, if ever, mentioned when I was a resident. We knew from clinical studies that children who were abused or neglected often develop severe mood or psychotic disorders in adulthood. But we did not know that trauma modifies some genes via under- or overexpression, and that such epigenetic changes alter brain development towards psychopathology. The mysteries of psychiatric brain disorders generated by childhood trauma have been clarified by advances in epigenetics.

Aspirational, futuristic therapies. Even now, as a seasoned psychiatric neuroscientist, I continue to dream.
Research is providing many clues for potentially radical psychiatric treatments that go beyond standard antipsychotics, antidepressants, mood stabilizers, or anxiolytics. But today, I fully expect that scientific dreams eventually come true through research. For example, the following neuroscientific therapeutics strategies may someday become routine in clinical practice:

- microglia inhibition

- mitochondria repair

- anti-apoptotic therapy

- white matter connectivity restoration

- neuroprotection (enhancing neurogenesis, increasing neurotropic factors, and enhancing synaptogenesis)

- reverse glutamate N-methyl-Daspartate hypofunction

- prevent amyloid formation.

Data analysis breakthroughs. Side-byside with the explosion of new findings and amassing mountains of data in psychiatric neuroscience, unprecedented and revolutionary data-management techniques have emerged to facilitate the herculean task of data analysis to extract the mythical needle in a haystack and derive the overall impact of masses of data. These techniques, whose names were not in our vocabulary during my residency days, include:

- machine learning

- artificial intelligence

- deep learning

- big data.

With the help of powerful computers and ingenious software, discovering critical nuggets of knowledge about the brain and predicting the best approaches to healing dysfunctional brains are now possible. Those powerful methods of analyzing massive data are the vehicles for transforming science fiction to reality by assembling the jigsaw puzzle(s) of the human brain, arguably the last frontier in medical science. 
From the Editor

continued from page 12

My life experiences as a psychiatric neuroscientist have convinced me that nothing is beyond the reach of scientific research. Unraveling the divine brain's complexities will eventually become reality. So, let us never stop dreaming and fantasizing!

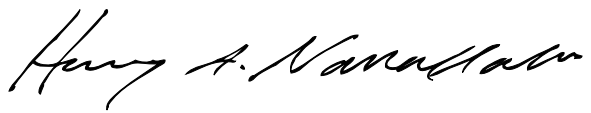

Henry A. Nasrallah, MD

Editor-in-Chief

References

1. Nasrallah HA, McCalley-Whitters M, Bigelow LB, et al. A histological study of the corpus callosum in chronic schizophrenia. Psychiatry Res. 1983;8(4):251-260.

2. Nasrallah HA, McCalley-Whitters M, Jacoby CG Cerebral ventricular enlargement in young manic males. A controlled CT study. J Affect Disord. 1982;4(1): 15-19.

3. Nasrallah HA, McCalley-Whitters M, Jacoby CG Cortical atrophy in schizophrenia and mania: a comparative CT study. J Clin Psychiatry. 1982;43(11) 439-441.

4. Nasrallah HA, Skinner TE, Schmalbrock P, et al. Proton magnetic resonance spectroscopy (1H MRS) of the hippocampal formation in schizophrenia: pilot study. Br J Psychiatry. 1994;165(4):481-485.

5. Nasrallah HA, Olson SC, McCalley-Whitters M, et al. Cerebral ventricular enlargement in schizophrenia. A preliminary follow-up study. Arch Gen Psychiatry. 1986;43(2):157-159. 